\title{
The synthesis of $\alpha$-benzotriazolyl ketones from acid halides
}

\author{
Alan R. Katritzky, ${ }^{* a}$ Kostyantyn Kirichenko, ${ }^{a}$ Prabhu P. Mohapatra, ${ }^{a}$ and Indra Prakash ${ }^{b}$ \\ ${ }^{a}$ Center for Heterocyclic Compounds, University of Florida, Department of Chemistry, \\ Gainesville, Florida 32611-7200, USA; \\ ${ }^{b}$ The NutraSweet Company, 1801 Maple Avenue, Evanston, IL 60201, USA \\ E-mail: Katritzky@chem.ufl.edu
}

\section{Dedicated to Professor Mieczyslaw Makosza on his $\mathbf{7 0}^{\text {th }}$ anniversary}

(received 06 Aug 03; accepted 29 Sept 03; published on the web 03 Oct 03)

\begin{abstract}
Reactions of benzotriazolylacetic acid 7 dianion with electrophiles gave 2benzotriazolylcarboxylic acids $9 \mathbf{a}-\mathbf{c}$. Dianions of $\mathbf{9 a}-\mathbf{c}$ were transformed with acid halides to $\alpha$ benzotriazolylketones $\mathbf{1 2} \mathbf{a}-\mathbf{i}$.
\end{abstract}

Keywords: $\alpha$-Benzotriazolyl ketones, acid halides, benzotriazolylacetic acid

\section{Introduction}

Ketones carrying a benzotriazolyl substituent in the $\alpha$-position to a carbonyl group, 1, are versatile intermediates (Scheme 1) for the further synthesis of (i-a) $\alpha$ - and (i-b) $\delta$ - diketones; ${ }^{1}$ (iia,b) $\alpha, \beta$-unsaturated ketones; ${ }^{2}$ (iii) olefins; ${ }^{3}$ (iv) acetylenes; ${ }^{4}$ (v) for the directed regioselective $\alpha$ alkylation of ketones; ${ }^{5}$ and (vi) in heterocycle ring synthesis. ${ }^{1 \mathrm{c}}$

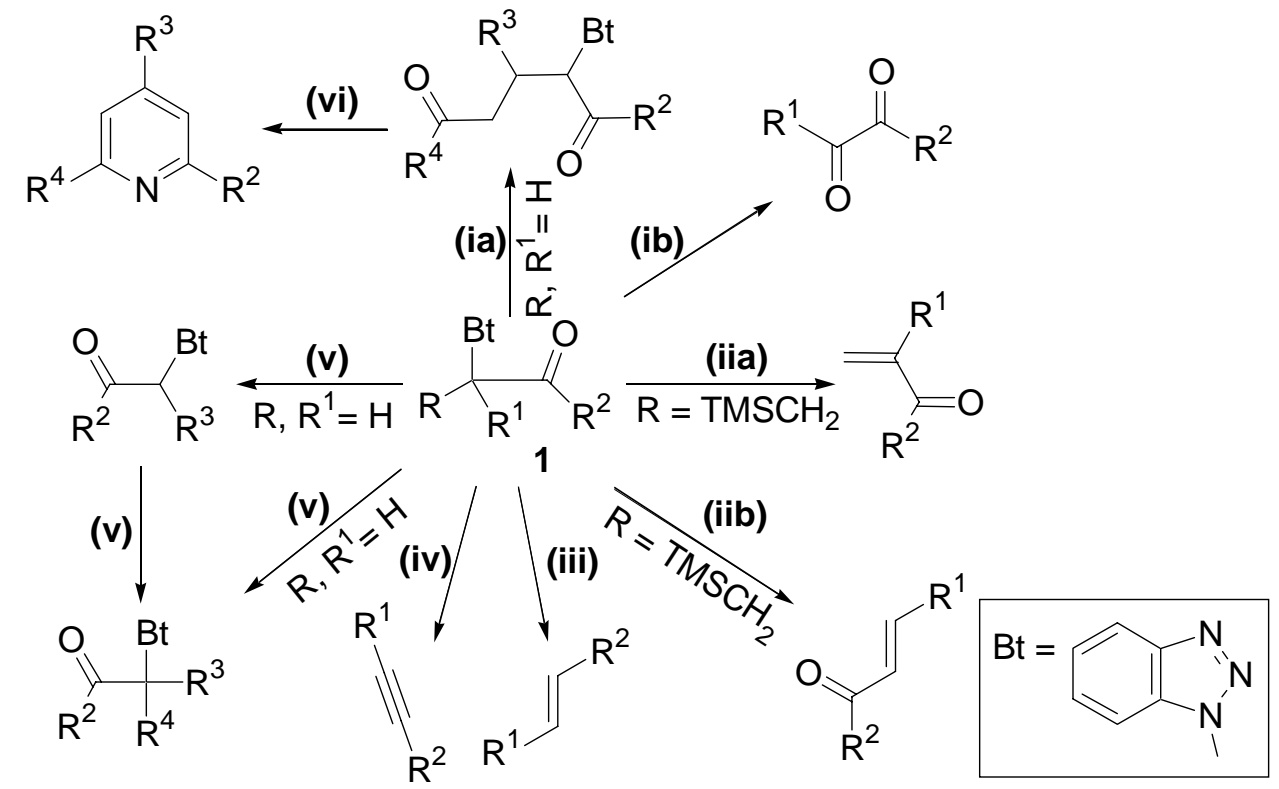

Scheme 1 
Available routes for the preparation of $\alpha$-benzotriazolyl ketones 1 (Scheme 2) comprise (i) reactions of carbanions from $N$-alkylbenzotriazoles 2 with appropriate esters or acid chlorides; ${ }^{1 \text { a- }}$ b,2,3,4,6 (ii) transformations of $\alpha$-benzotriazolylalkylcarboxylic esters 3 with Grignard reagents; ${ }^{4}$ (iii) from $\alpha$-haloketones $4 ;^{7}$ (iv) addition of benzotriazole to but-2-ene-1,4-diones $\mathbf{5} ;^{8}$ and (v) oxidation of $\alpha$-benzotriazolyl secondary alcohols $6^{9}$ (Scheme 2 ).

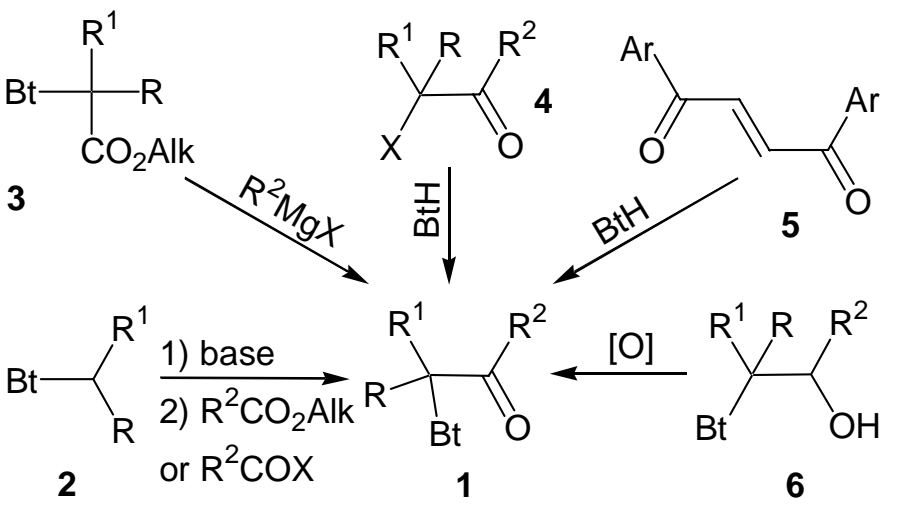

\section{Scheme 2}

We have now established the capability of the benzotriazolyl group in 2-benzotriazolylacetic acid 7 to stabilize the dianions in successive subsequent reactions with alkyl halides $\mathbf{8 a}-\mathbf{c}$ and acid halides 10a-d and then to induce decarboxylation to give $\alpha$-benzotriazolyl ketones 12a-i (Scheme 3). The new transformations now reported of acid halides with dianions of 2benzotriazolylcarboxylic acids $9 \mathbf{a}-\mathbf{c}$, derived from 2-benzotriazolylacetic acid 7, provide a facile route to diverse $\alpha$-benzotriazolyl ketones $\mathbf{1 2 a}-\mathbf{i}$ in good overall yields.

\section{Results and Discussion}

Benzotriazol-1-ylacetic acid 7 was easily prepared from chloroacetic acid and benzotriazole according to published procedure in $60 \%$ yield. ${ }^{10}$ The treatment of benzotriazol-1-ylacetic acid 7 with $n$-butyllithium (2.2 eq.) in THF at $-78{ }^{\circ} \mathrm{C}$ followed by the reaction with electrophiles 8a-c (1.3-1.5 eq.) gave intermediates $9 \mathbf{a}-\mathbf{c}$ in $50-89 \%$ yields (Scheme 3, Table 1). The structures of compounds 9a-c were supported by their ${ }^{1} \mathrm{H}$ NMR and ${ }^{13} \mathrm{C}$ NMR spectra.

The compounds 9a-c were dilithiated with $n$-butyllithium $\left(2.2\right.$ eq.) at $-78{ }^{\circ} \mathrm{C}$ for $2 \mathrm{~h}$ (deep red color of reaction mixture) followed by addition of corresponding acid chlorides 10a-d (1.01.1 eq.) at the same temperature to give intermediate lithium salts $\mathbf{1 1}$. The decarboxylation of $\mathbf{1 1}$ upon acidification of the reaction mixture with mineral acid (hydrochloric or sulfuric) to $\mathrm{pH}=4-5$ gave $\alpha$-benzotriazolyl ketones 12a-i in $60-84 \%$ overall yields. The attempted preparation of ketone 12a using methyl 4-methylbenzoate, as an electrophile instead of 4-methylbenzoyl chloride 10b, gave ketone 12a in only 30\% yield. The structures of ketones 12a-i were supported by their ${ }^{1} \mathrm{H}$ NMR and ${ }^{13} \mathrm{C}$ NMR spectra. The ${ }^{13} \mathrm{C}$ NMR spectra of $\mathbf{1 2} \mathbf{a}-\mathbf{i}$ no longer show carbon signals in the 169.9-170.9 ppm range, which corresponds to the carboxyl group in 
9a-c. For 12a-i, new signals in 189.9-202.9 ppm range were assigned to the carbonyl group of $\alpha$-benzotriazolyl ketones $\mathbf{1 2 a}-\mathbf{i}$.

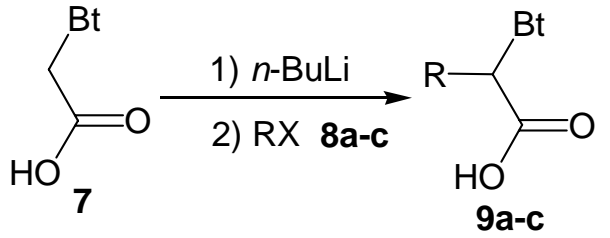

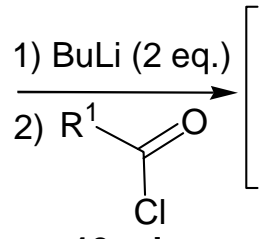<smiles>[R]C(=O)C([R])(Br)C(=O)OC</smiles>

8a: $\mathrm{CH}_{3} \mathrm{l}$

8b: AllylBr 8c: $4-\mathrm{CH}_{3} \mathrm{C}_{6} \mathrm{H}_{4} \mathrm{CH}_{2} \mathrm{Br}$

10a: $\mathrm{R}^{1}=\mathrm{CH}_{3}$ 10b: $\mathrm{R}^{1}=4-\mathrm{CH}_{3} \mathrm{C}_{6} \mathrm{H}_{4}$

10c: $R^{1}=2$-furyl 10d: $R^{1}=B n$<smiles>[R]C(=O)C([R])Br</smiles>

Scheme 3. For designation of $R$ and $R^{1}$ in $9 \mathbf{a}-\mathbf{c}, \mathbf{1 1}$, and 12a-i see Table 1 .

Table 1. Synthesis of $\alpha$-benzotriazolylcarboxylic acids $9 \mathbf{a}-\mathbf{c}$ and $\alpha$-benzotriazolyl ketones 12a-i

\begin{tabular}{|c|c|c|c|}
\hline Compounds & $\mathrm{R}$ & $\mathrm{R}^{1}$ & Yields, \% \\
\hline $9 \mathbf{a}$ & $\mathrm{Me}$ & - & $50-65$ \\
\hline $9 b$ & Allyl & - & 65 \\
\hline $9 \mathrm{c}$ & 4- $\mathrm{CH}_{3} \mathrm{C}_{6} \mathrm{H}_{4} \mathrm{CH}_{2}$ & - & 89 \\
\hline $12 \mathbf{a}^{4,12}$ & $\mathrm{H}$ & $4-\mathrm{CH}_{3} \mathrm{C}_{6} \mathrm{H}_{4}$ & $56\left(30^{\mathrm{a}}\right)$ \\
\hline $12 b^{12}$ & $\mathrm{H}$ & $\mathrm{Bn}$ & 60 \\
\hline $12 \mathrm{c}$ & $\mathrm{Me}$ & $\mathrm{Me}$ & 61 \\
\hline $12 d^{12}$ & $\mathrm{Me}$ & 4- $\mathrm{CH}_{3} \mathrm{C}_{6} \mathrm{H}_{4}$ & 80 \\
\hline $12 \mathrm{e}$ & $\mathrm{Me}$ & 2-Furyl & 61 \\
\hline $12 f$ & Allyl & $\mathrm{Me}$ & 60 \\
\hline $12 \mathrm{~g}$ & Allyl & $4-\mathrm{CH}_{3} \mathrm{C}_{6} \mathrm{H}_{4}$ & 60 \\
\hline $12 \mathrm{~h}$ & $4-\mathrm{CH}_{3} \mathrm{C}_{6} \mathrm{H}_{4} \mathrm{CH}_{2}$ & $\mathrm{Me}$ & 84 \\
\hline $12 \mathrm{i}$ & $4-\mathrm{CH}_{3} \mathrm{C}_{6} \mathrm{H}_{4} \mathrm{CH}_{2}$ & $4-\mathrm{CH}_{3} \mathrm{C}_{6} \mathrm{H}_{4}$ & 79 \\
\hline
\end{tabular}

${ }^{a}$ Methyl 4-methylbenzoate was used instead of 4-methylbenzoyl chloride.

In summary, a novel and efficient procedure was developed for the preparation of $\alpha$ benzotriazolyl ketones. 


\section{Experimental Section}

General Procedures. All melting points were determined on a Mel-Temp II melting point apparatus and are uncorrected. NMR spectra were recorded on a Varian Gemini 300 spectrometer in chloroform- $d$ and DMSO- $d_{6}$ with TMS as internal reference for ${ }^{1} \mathrm{H}(300 \mathrm{MHz})$ or a solvent as the internal reference for ${ }^{13} \mathrm{C}(75 \mathrm{MHz})$. The elemental analyses were performed on a Carlo Erba EA-1108 instrument. THF was dried over sodium / benzophenone and used freshly distilled. Column chromatography was conducted on silica gel 200-425 meshes. All of the chemicals were employed as supplied.

\section{General procedure for the preparation of $\alpha$-benzotriazolylalkylcarboxylic acids 9a-c}

A solution of $n$-butyllithium $(6.9 \mathrm{~mL}, 11.0 \mathrm{mmol}, 1.6 \mathrm{M}$ in hexane) was added dropwise to a stirred solution of benzotriazolylacetic acid $7(0.89 \mathrm{~g}, 5 \mathrm{mmol})$ in THF $(30 \mathrm{~mL})$ at $-78^{\circ} \mathrm{C}$. The reaction mixture was stirred at this temperature for $2 \mathrm{~h}$ and corresponding $8 \mathbf{8}-\mathbf{c}(7.5 \mathrm{mmol})$ in THF $(10 \mathrm{~mL})$ was added dropwise to the reaction mixture. The reaction mixture was stirred at $78{ }^{\circ} \mathrm{C}$ for $2 \mathrm{~h}$ and then for an additional $2 \mathrm{~h}$ at $20-25^{\circ} \mathrm{C}$. An aqueous sodium hydroxide solution $(3 \%, 30 \mathrm{~mL})$ was added to the reaction mixture and the mixture was washed with diethyl ether $(3 \times 30 \mathrm{~mL})$. Diethyl ether remaining in the aqueous layer was removed in vacuum and the aqueous layer was acidified with concentrated hydrochloric acid to $\mathrm{pH}=3-4$. The precipitate was filtered off, washed with cold water $(20-30 \mathrm{~mL})$ and dried in vacuum to give pure 9a-c.

2-(1H-1,2,3-Benzotriazol-1-yl)propionic acid (9a). White microcrystals from diethyl ether (50$65 \%$ ), mp 181-182 ${ }^{\circ} \mathrm{C}$ (lit. ${ }^{11} \mathrm{mp} 179-181{ }^{\circ} \mathrm{C}$ ). ${ }^{1} \mathrm{H}$ NMR (DMSO- $\left.d_{6}\right): \delta=8.09-8.06(\mathrm{~m}, 1 \mathrm{H})$, 7.86-7.83 (m, 1H), 7.58-7.53 (m, 1H), 7.44-7.39 (m, 1H), 5.98 (q, $J=7.2 \mathrm{~Hz}, 1 \mathrm{H}), 1.93(\mathrm{~d}, J=$ $7.2 \mathrm{~Hz}, 3 \mathrm{H}$ ). ${ }^{13} \mathrm{C}$ NMR (DMSO- $\left.d_{6}\right): \delta=170.9,145.2,133.0,127.4,124.0,119.2,110.9,56.0$, 16.5. Anal. Calcd. for $\mathrm{C}_{9} \mathrm{H}_{9} \mathrm{~N}_{3} \mathrm{O}_{2}$ (191.19): C, 56.54; H, 4.74; N, 21.98. Found: C, 56.84; H, 4.80; $\mathrm{N}, 22.04$.

2-(1H-1,2,3-Benzotriazol-1-yl)pent-4-enoic acid (9b). White microcrystals from diethyl ether $(65 \%), \mathrm{mp} 165-166{ }^{\circ} \mathrm{C} .{ }^{1} \mathrm{H}$ NMR (DMSO- $\left.d_{6}\right): \delta=8.06(\mathrm{~d}, J=8.4 \mathrm{~Hz}, 1 \mathrm{H}), 7.87(\mathrm{~d}, J=8.2 \mathrm{~Hz}$, $1 \mathrm{H}), 7.58-7.53(\mathrm{~m}, 1 \mathrm{H}), 7.44-7.38(\mathrm{~m}, 1 \mathrm{H}), 6.00(\mathrm{dd}, J=9.6,5.6 \mathrm{~Hz}, 1 \mathrm{H}), 5.74-5.60(\mathrm{~m}, 1 \mathrm{H})$, $4.94(\mathrm{~d}, J=17.2 \mathrm{~Hz}, 1 \mathrm{H}), 4.86(\mathrm{~d}, J=10.2 \mathrm{~Hz}, 1 \mathrm{H}), 3.20-3.14(\mathrm{~m}, 2 \mathrm{H}) .{ }^{13} \mathrm{C}$ NMR (DMSO- $\left.d_{6}\right): \delta$ 169.9, 145.2, 133.3, 133.1, 127.5, 124.0, 119.3, 118.5, 110.9, 60.1, 34.4. Anal. Calcd. for $\mathrm{C}_{11} \mathrm{H}_{11} \mathrm{~N}_{3} \mathrm{O}_{2}$ (217.23): C, 60.82; H, 5.10; N, 19.34. Found: C, 61.13; H, 5.21; N, 19.31.

2-(1H-1,2,3-Benzotriazol-1-yl)-3-(4-methylphenyl)propionic acid (9c). White microcrystals from diethyl ether $(89 \%), \mathrm{mp} 208-209{ }^{\circ} \mathrm{C} .{ }^{1} \mathrm{H}$ NMR (DMSO- $\left.d_{6}\right): \delta=7.99(\mathrm{~d}, J=8.3 \mathrm{~Hz}, 1 \mathrm{H})$, $7.82(\mathrm{~d}, J=8.3 \mathrm{~Hz}, 1 \mathrm{H}), 7.51-7.46(\mathrm{~m}, 1 \mathrm{H}), 7.35(\mathrm{t}, J=7.5 \mathrm{~Hz}, 1 \mathrm{H}), 6.99(\mathrm{~d}, J=7.9 \mathrm{~Hz}, 2 \mathrm{H})$, $6.90(\mathrm{~d}, J=7.9 \mathrm{~Hz}, 2 \mathrm{H}), 6.21(\mathrm{dd}, J=9.0,7.1 \mathrm{~Hz}, 1 \mathrm{H}), 3.72(\mathrm{~d}, J=8.6 \mathrm{~Hz}, 2 \mathrm{H}), 2.11(\mathrm{~s}, 3 \mathrm{H})$.

${ }^{13} \mathrm{C}$ NMR (DMSO- $d_{6}$ ): $\delta=169.9,145.0,135.6,133.4,133.4,128.8,128.6,127.4,123.9,119.1$, 110.8, 61.8, 35.5, 20.5. Anal. Calcd. for $\mathrm{C}_{16} \mathrm{H}_{15} \mathrm{~N}_{3} \mathrm{O}_{2}$ (281.32): C, 68.31; H, 5.37; N, 14.94 . Found: C, 67.96; H, 5.32; N, 14.90 .

\section{General procedure for the preparation of $\alpha$-benzotriazolyl ketones 12a-i}

A solution of $n$-butyllithium $(2.45 \mathrm{~mL}, 3.9 \mathrm{mmol}, 1.6 \mathrm{M}$ in hexane, $)$ was added dropwise to a stirred solution of 7 or $9 \mathbf{a}-\mathbf{c}(1.8 \mathrm{mmol})$ in THF $(30 \mathrm{~mL})$ at $-78^{\circ} \mathrm{C}$. The reaction mixture was 
stirred at this temperature for $2 \mathrm{~h}$ and corresponding acid chloride 10a-d (1.9 mmol) was added dropwise. The reaction mixture was stirred at $-78^{\circ} \mathrm{C}$ for $15-30 \mathrm{~min}$ and then for an additional 15 min at $20-25^{\circ} \mathrm{C}$. A diluted hydrochloric acid was added to the reaction mixture to adjust $\mathrm{pH}=5$ and product was extracted with diethyl ether $(2 \times 50 \mathrm{~mL})$. The extract was concentrated in vacuum and the product $\mathbf{1 2} \mathbf{a}-\mathbf{i}$ was purified by column chromatography or recrystallized.

2-(1H-1,2,3-Benzotriazol-1-yl)-1-(4-methylphenyl)ethanone (12a). White microcrystals from toluene (56\%), mp 132-134 ${ }^{\circ} \mathrm{C}$ (lit. $\left.{ }^{4,12} \mathrm{mp} 133-135{ }^{\circ} \mathrm{C}\right) .{ }^{1} \mathrm{H} \mathrm{NMR}\left(\mathrm{CDCl}_{3}\right): \delta=8.09(\mathrm{~d}, J=8.2$ $\mathrm{Hz}, 1 \mathrm{H}), 7.96(\mathrm{~d}, J=8.2 \mathrm{~Hz}, 2 \mathrm{H}), 7.51-7.33(\mathrm{~m}, 5 \mathrm{H}), 6.08(\mathrm{~s}, 2 \mathrm{H}), 2.46(\mathrm{~s}, 3 \mathrm{H}) .{ }^{13} \mathrm{C} \mathrm{NMR}$ $\left(\mathrm{CDCl}_{3}\right): \delta=189.9,146.1,145.7,133.8,131.5,129.8,128.4,127.8,124.0,120.1,109.6,53.8$, 21.8 .

1-(1H-1,2,3-Benzotriazol-1-yl)-3-phenyl-propan-2-one (12b). White microcrystals from diethyl ether $(60 \%)$, mp $141-142{ }^{\circ} \mathrm{C}$ (lit. $\left.{ }^{12} \mathrm{mp} 141-142{ }^{\circ} \mathrm{C}\right) .{ }^{1} \mathrm{H} \mathrm{NMR}\left(\mathrm{CDCl}_{3}\right): 8.03(\mathrm{~d}, J=8.2 \mathrm{~Hz}, 1 \mathrm{H})$, 7.39-7.26 (m, 8H), $5.43(\mathrm{~s}, 2 \mathrm{H}), 3.78$ (s, 2H.). ${ }^{13} \mathrm{C} \mathrm{NMR}\left(\mathrm{CDCl}_{3}\right): \delta=199.7,146.0,133.5$, $132.3,129.4,129.2,127.9,127.8,124.2,120.1,109.2,55.5,47.4$.

3-(1H-1,2,3-Benzotriazol-1-yl)butan-2-one (12c). Colorless oil (61\%, column chromatography with ethyl acetate/hexanes 1:9). ${ }^{1} \mathrm{H}$ NMR $\left(\mathrm{CDCl}_{3}\right): \delta=8.09(\mathrm{~d}, J=8.2 \mathrm{~Hz}, 1 \mathrm{H}), 7.50(\mathrm{~d}, J=3.8$ $\mathrm{Hz}, 2 \mathrm{H}), 7.42-7.37(\mathrm{~m}, 1 \mathrm{H}), 5.67(\mathrm{q}, J=7.3 \mathrm{~Hz}, 1 \mathrm{H}), 2.06(\mathrm{~s}, 3 \mathrm{H}), 1.93(\mathrm{~d}, J=7.3 \mathrm{~Hz}, 3 \mathrm{H}) .{ }^{13} \mathrm{C}$ $\operatorname{NMR}\left(\mathrm{CDCl}_{3}\right): \delta=202.9,146.0,132.5,127.7,124.2,120.0,109.5,63.3,26.1,15.4$. Anal. Calcd. for $\mathrm{C}_{10} \mathrm{H}_{11} \mathrm{~N}_{3} \mathrm{O}$ (189.22): C, 63.48; H, 5.86; N, 22.21. Found: C, 63.68; H, 5.57; N, 22.18.

2-(1H-1,2,3-Benzotriazol-1-yl)-1-(4-methylphenyl)propan-1-one (12d). Colorless oil ${ }^{12}$ (80\%, column chromatography with ethyl acetate/hexanes 1:3). ${ }^{1} \mathrm{H}$ NMR $\left(\mathrm{CDCl}_{3}\right): \delta=8.01(\mathrm{~d}, J=8.4$ $\mathrm{Hz}, 1 \mathrm{H}), 7.90(\mathrm{~d}, J=8.1 \mathrm{~Hz}, 2 \mathrm{H}), 7.53(\mathrm{~d}, J=8.2 \mathrm{~Hz}, 1 \mathrm{H}), 7.42-7.37(\mathrm{~m}, 1 \mathrm{H}), 7.31-7.26(\mathrm{~m}$, $1 \mathrm{H}), 7.16(\mathrm{~d}, J=8.1 \mathrm{~Hz}, 2 \mathrm{H}), 6.70(\mathrm{q}, J=7.1 \mathrm{~Hz}, 1 \mathrm{H}), 2.29(\mathrm{~s}, 3 \mathrm{H}), 1.95(\mathrm{~d}, J=7.1 \mathrm{~Hz}, 3 \mathrm{H}) .{ }^{13} \mathrm{C}$ $\operatorname{NMR}\left(\mathrm{CDCl}_{3}\right): \delta=193.2,146.2,144.9,131.9,131.4,129.4,128.6,127.4,123.8,119.8,110.2$, $59.1,21.4,16.1$.

2-(1H-1,2,3-Benzotriazol-1-yl)-1-(furan-2-yl)propan-1-one (12e). Colorless oil (61\%, column chromatography with ethyl acetate/hexanes $1: 9) .{ }^{1} \mathrm{H} \mathrm{NMR}\left(\mathrm{CDCl}_{3}\right): \delta=8.04(\mathrm{~d}, J=8.2 \mathrm{~Hz}, 1 \mathrm{H})$, 7.61-7.57 (m, 2H), 7.45 (t, $J=7.5 \mathrm{~Hz}, 1 \mathrm{H}), 7.37-7.32(\mathrm{~m}, 1 \mathrm{H}), 7.26(\mathrm{~d}, J=3.7 \mathrm{~Hz}, 1 \mathrm{H}), 6.50$ $6.40(\mathrm{~m}, 2 \mathrm{H}), 2.01(\mathrm{~d}, J=7.2 \mathrm{~Hz}, 3 \mathrm{H}) .{ }^{13} \mathrm{C} \mathrm{NMR}\left(\mathrm{CDCl}_{3}\right): \delta=182.5,150.2,147.7,146.5,132.4$, 127.7, 124.1, 120.1, 119.9, 112.9, 110.5, 59.7, 15.9. Anal. Calcd. for $\mathrm{C}_{13} \mathrm{H}_{11} \mathrm{~N}_{3} \mathrm{O}_{2}$ (241.25): $\mathrm{C}$, 64.72; H, 4.60; N, 17.42. Found: C, 64.51; H, 4.82; N, 16.78.

3-(1H-1,2,3-Benzotriazol-1-yl)hex-5-en-2-one $\quad$ (12f). Colorless oil $\quad(60 \%$, column chromatography with ethyl acetate/hexanes 1:9). ${ }^{1} \mathrm{H}$ NMR $\left(\mathrm{CDCl}_{3}\right): \delta=8.10(\mathrm{~d}, J=8.3 \mathrm{~Hz}, 1 \mathrm{H})$, $7.52(\mathrm{~d}, J=3.9 \mathrm{~Hz}, 1 \mathrm{H}), 7.44-7.39(\mathrm{~m}, 2 \mathrm{H}), 5.70-5.53(\mathrm{~m}, 2 \mathrm{H}), 5.05-4.93(\mathrm{~m}, 2 \mathrm{H}), 3.22-3.10$ $(\mathrm{m}, 2 \mathrm{H}), 2.06(\mathrm{~s}, 3 \mathrm{H}) .{ }^{13} \mathrm{C} \mathrm{NMR}\left(\mathrm{CDCl}_{3}\right): \delta=202.2,146.2,132.9,131.9,128.0,124.3,120.3$, 119.3, 109.6, 67.6, 33.8, 26.9. Anal. Calcd. for $\mathrm{C}_{12} \mathrm{H}_{13} \mathrm{~N}_{3} \mathrm{O}$ (215.26): C, 66.96; H, 6.09; N, 19.52. Found: C, 66.69; H, 6.24; N, 19.63 .

2-(1H-1,2,3-Benzotriazol-1-yl)-1-(4-methylphenyl)pent-4-en-1-one (12g). Colorless oil (60\%, column chromatography with ethyl acetate/hexanes 1:9). ${ }^{1} \mathrm{H}$ NMR $\left(\mathrm{CDCl}_{3}\right): \delta=8.00(\mathrm{~d}, J=8.3$ $\mathrm{Hz}, 1 \mathrm{H}), 7.92(\mathrm{~d}, J=8.2 \mathrm{~Hz}, 2 \mathrm{H}), 7.62(\mathrm{~d}, J=8.4 \mathrm{~Hz}, 1 \mathrm{H}), 7.45-7.40(\mathrm{~m}, 1 \mathrm{H}), 7.33-7.28(\mathrm{~m}$, $1 \mathrm{H}), 7.17(\mathrm{~d}, J=8.2 \mathrm{~Hz}, 2 \mathrm{H}), 6.65(\mathrm{dd}, J=8.7,6.4 \mathrm{~Hz}, 1 \mathrm{H}), 5.77-5.64(\mathrm{~m}, 1 \mathrm{H}), 5.02-4.94(\mathrm{~m}$, 2H), 3.21-3.10 (m, 2H), $2.30(\mathrm{~s}, 3 \mathrm{H}) .{ }^{13} \mathrm{C} \mathrm{NMR}\left(\mathrm{CDCl}_{3}\right): \delta=192.5,146.6,145.3,132.2,132.1$, 
132.0, 129.7, 129.0, 127.8, 124.1, 120.2, 119.3, 110.7, 63.2, 34.3, 21.7. Anal. Calcd. for $\mathrm{C}_{18} \mathrm{H}_{17} \mathrm{~N}_{3} \mathrm{O}$ (291.36): C, 74.20; H, 5.88; N, 14.42. Found: C, 73.81; H, 5.92; N, 14.38.

3-(1H-1,2,3-Benzotriazol-1-yl)-4-(4-methylphenyl)butan-2-one (12h). Colorless oil (84\%, column chromatography with ethyl acetate/hexanes 1:9). ${ }^{1} \mathrm{H}$ NMR $\left(\mathrm{CDCl}_{3}\right): \delta=8.03(\mathrm{~d}, J=8.1$ $\mathrm{Hz}, 1 \mathrm{H}), 7.44-7.31(\mathrm{~m}, 3 \mathrm{H}), 6.90(\mathrm{~d}, J=8.1 \mathrm{~Hz}, 2 \mathrm{H}), 6.85(\mathrm{~d}, J=8.1 \mathrm{~Hz}, 2 \mathrm{H}), 5.65(\mathrm{dd}, J=$ 10.0, $5.2 \mathrm{~Hz}, 1 \mathrm{H}), 3.72(\mathrm{dd}, J=14.3,5.2 \mathrm{~Hz}, 1 \mathrm{H}), 3.52(\mathrm{dd}, J=14.3,10.0 \mathrm{~Hz}, 1 \mathrm{H}), 2.16(\mathrm{~s}, 3 \mathrm{H})$, $2.02(\mathrm{~s}, 3 \mathrm{H}) .{ }^{13} \mathrm{C} \mathrm{NMR}\left(\mathrm{CDCl}_{3}\right): \delta=202.2,146.0,136.6,133.0,132.8,129.3,128.6,127.9$, 124.2, 120.2, 109.5, 69.5, 35.4, 27.1, 20.9. Anal. Calcd. for $\mathrm{C}_{17} \mathrm{H}_{17} \mathrm{~N}_{3} \mathrm{O}$ (279.34): C, 73.10; $\mathrm{H}$, $6.13 ; \mathrm{N}, 15.04$. Found: C, 73.10; H, 6.29; N, 14.94 .

2-(1H-1,2,3-Benzotriazol-1-yl)-1,3-bis(4-methylphenyl)propan-1-one (12i). White microcrystals from diethyl ether/hexanes $(79 \%), \mathrm{mp} 135-136{ }^{\circ} \mathrm{C} .{ }^{1} \mathrm{H} \mathrm{NMR}\left(\mathrm{CDCl}_{3}\right): \delta=7.98(\mathrm{~d}, J=8.4 \mathrm{~Hz}$, $1 \mathrm{H}), 7.89$ (d, $J=8.2 \mathrm{~Hz}, 2 \mathrm{H}), 7.60$ (d, $J=8.4 \mathrm{~Hz}, 1 \mathrm{H}), 7.46-7.41(\mathrm{~m}, 1 \mathrm{H}), 7.34-7.29(\mathrm{~m}, 1 \mathrm{H})$, $7.15(\mathrm{~d}, J=8.1 \mathrm{~Hz}, 2 \mathrm{H}), 6.95(\mathrm{~d}, J=8.0 \mathrm{~Hz}, 2 \mathrm{H}), 6.87(\mathrm{~d}, J=8.0 \mathrm{~Hz}, 2 \mathrm{H}), 6.72(\mathrm{dd}, J=9.0,6.0$ Hz, 1H), 3.74 (dd, $J=14.3,6.0 \mathrm{~Hz}, 1 \mathrm{H}), 3.57$ (dd, $J=14.3,9.0 \mathrm{~Hz}, 1 \mathrm{H}), 2.31$ (s, 3H), $2.22(\mathrm{~s}$, $3 \mathrm{H}) .{ }^{13} \mathrm{C} \mathrm{NMR}\left(\mathrm{CDCl}_{3}\right): \delta=192.6,146.6,145.4,136.9,133.1,132.4,132.2,129.8,129.5,129.1$, 129.0, 127.9, 124.2, 120.4, 110.8, 65.2, 35.9, 21.8, 21.1. Anal. Calcd. for $\mathrm{C}_{23} \mathrm{H}_{21} \mathrm{~N}_{3} \mathrm{O}(355.44)$ : C, 77.72; H, 5.96; N, 11.82. Found: C, 77.72; H, 6.08; N, 11.94.

\section{References}

1. (a) Katritzky, A. R.; Kuzmierkiewicz, W. J. Chem. Soc., Perkin Trans. 1 1987, 819. (b) Katritzky, A. R.; Wang, Z.; Lang, H.; Feng, D. J. Org. Chem. 1997, 62, 4125. (c) Katritzky, A. R.; Abdel-Fattah, A. A. A.; Tymoshenko, D. O.; Essawy, S. A. Synthesis 1999, 2114. (d) Faust, R.; Weber, C. US Pat. 6121442, 2000; Ger. Pat. DE 19626120, 1998; Chem. Abstr. 1998, 128, 114783.

2. (a) Katritzky, A. R.; Voronkov, M. V.; Toader, D. J. Chem. Soc., Perkin Trans. 2 1998, 2515. (b) Katritzky, A. R.; Toader, D.; Chassaing, C. J. Org. Chem. 1998, 63, 9983.

3. (a) Katritzky, A. R.; Fali, C. N.; Li, J. J. Org. Chem. 1997, 62, 4148. (b) Katritzky, A. R.; Cheng, D.; Li, J. J. Org. Chem. 1998, 63, 3438.

4. Katritzky, A. R.; Wang, J.; Karodia, N.; Li, J. J. Org. Chem. 1997, 62, 4142.

5. Katritzky, A. R.; Wang, Z.; Ji, Y.; Fang Y. ARKIVOC 2002, (iii), 46.

6. (a) Katritzky, A. R.; Lan, X.; Lam, J. N. Chem. Ber. 1991, 124, 1819. (b) Katritzky, A. R.; Lan, X.; Lam, J. N. J. Org. Chem. 1991, 56, 4397. (c) Katritzky, A. R.; Rachwal, S.; Hitchings, G. J. Tetrahedron 1991, 47, 2683. (d) Katritzky, A. R.; Yao, G.; Rachwal, S. J. Heterocycl. Chem. 1994, 31, 757. (e) Katritzky, A. R.; Zhang, G.; Jiang, J. J. Org. Chem. 1995, 60, 7605. (f) Katritzky, A. R.; Lang, H. J. Org. Chem. 1995, 60, 7612. (g) Katritzky, A. R.; Toader, D. J. Am. Chem. Soc. 1997, 119, 9321. (h) Katritzky, A. R.; Wang, J.; Henderson, S. A. Heterocycles 1998, 48, 1567.

7. (a) Katritzky, A. R.; Wu, J.; Wrobel, L.; Rachwal, S.; Steel, P. J. Acta. Chem. Scand. 1993, 47, 167. (b) Al-Omran, F.; Al-Awadl, N.; Yousef, O.; Elnagdi, M. H. J. Heterocycl. Chem. 2000, 37, 167.

8. Katritzky, A. R.; Rachwal, S.; Hughes, C. V.; Wang, Z. Pol. J. Chem. 1992, 66, 1633. 
9. Faust, R.; Weber, C. Liebigs Ann. 1996, 1235.

10. Krollpfeiffer, F.; Rosenberg, A.; Mühlhausen, C. Liebigs Ann. Chem. 1935, 515, 113.

11. Sparatore, F.; La Rotonda, M. I.; Caliendo, G.; Novellino, E.; Silipo, C.; Vittoria, A. Farmaco, Ed. Sci. 1988, 43, 29; Chem. Abstr. 1988, 109, 128916.

12. Katritzky, A. R.; Lam, J. N. Heteroatom Chem. 1990, 1, 21. 